\title{
Improved spectral aberration in Johnson Noise Thermometry
}

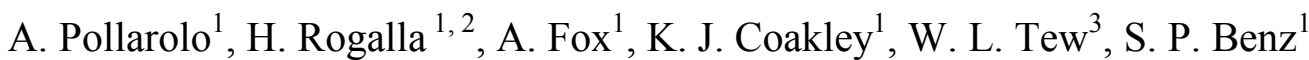 \\ ${ }^{1}$ National Institute of Standards and Technology, Boulder, CO, USA \\ alessio.pollarolo@nist.gov \\ ${ }^{2}$ ECEE Department, University of Colorado at Boulder, Boulder, CO, USA \\ ${ }^{3}$ National Institute of Standards and Technology, Gaithersburg, MD, USA
}

\begin{abstract}
Spectral aberration has been the main source of uncertainty in Johnson Noise Thermometry approach to measuring the Boltzmann constant. Recently, with newly developed hardware and the introduction of a novel fitting algorithm for analyzing the data, we have achieved a frequency independent spectral aberration for measurements with the NIST JNT system. Consequently, we performed a data drift analysis and an electromagnetic interference investigation to explain a residual offset that affects the Boltzmann constant.
\end{abstract}

Index Terms - Boltzmann equation, Josephson junction, measurement units, noise measurement, standards, temperature measurement.

\section{INTRODUCTION}

Johnson noise is the thermal fluctuation of the electrons in an electrical resistor. The Nyquist relation describes this phenomenon such that the time-averaged mean-square voltage $\left\langle V^{2}\right\rangle$ across a resistance $R$ is

$$
\left\langle V^{2}\right\rangle=4 k T R \Delta f .
$$

Here $k$ is the Boltzmann constant, $T$ is the resistor temperature, and $\Delta f$ is the measurement bandwidth.

Since 2002, NIST has been operating and developing Johnson Noise Thermometry systems based on a Quantum Voltage Noise Source (QVNS) [1]. These systems allow a direct electronic method to determine the Boltzmann constant when they are optimized to measure the noise of a sense resistor at the triple point of water (TPW).

A cross-correlation measurement is necessary to minimize the effect of uncorrelated noise due to voltage amplifiers. The voltage noise of each source, namely, the QVNS and the sense resistor, is alternatively measured for $100 \mathrm{~s}$ with two lownoise amplifier channels, from which is calculated the autoand cross-correlation.

\section{FitTING SELECTION MODEL}

The major system limitation has been the bandwidth dependence of the ratio of the measured power spectral density of the two noise sources. For comparison, we calculate the "offset" $\left(a_{0}-a_{0 \mathrm{calc}}\right)$, which correspond to the $k$, for different bandwidths, namely, the difference of the $a_{0}$ intercept of the cross-correlation ratio of the spectral density of the two noise sources (after fitting over a chosen bandwidth) from the calculated value.

Recently, we introduced a new approach to determine the Boltzmann constant based on a cross-validation method [2].

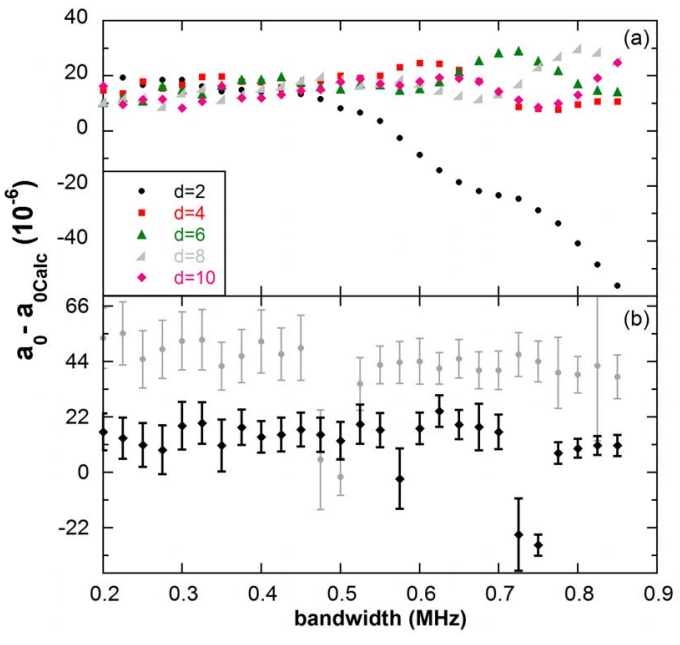

Fig. 1. Cross-correlation ratio fitting. (a) Spectral aberration obtained with higher order "d" polynomials as a function of measurement bandwidth for data "set 2". (b) Model selection results for an older data "set 1" (gray) and the newest data "set 2" (black).

The cross-correlation ratio is fit with an even polynomial function whose complexity (polynomial order) is selected after determining whether the given model is consistent with validation data and training data. The model selection algorithm determines the optimum model or order as a function of the fitting bandwidth. This method was applied to various data sets that were obtained under different conditions.

In Fig. 1, we report the results of the method applied to our newest data "set 2," which was optimally obtained under stable conditions and in a low electromagnetic interference (EMI) environment, whereas "set 1 " was obtain in a common laboratory. Fig. 1(a) reports the offsets vs. different bandwidths that were obtained for five different models with fixed complexity. Fig. 1(b) shows for old and new data sets the selected offsets for different bandwidths that were determined using the model selection algorithm Thanks to hardware improvements [3] and the new model selection method, we achieved ("set 2") for the first time nearly bandwidth independent results between $200 \mathrm{kHz}$ and $700 \mathrm{kHz}$ for the determination of $k$, given the reported uncertainty.

\section{DATA DRIFT AND EMI INVESTIGATION}

In order to search for systematic errors, we applied the model selection method to the daily single measurements that made up the above data set and fixed the analysis bandwidth 


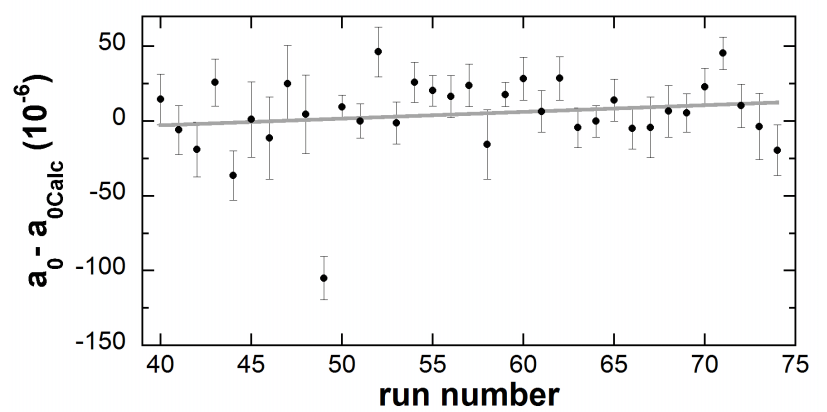

Fig. 2. Values of $\mathrm{a}_{0}-\mathrm{a}_{0 \text { Calc }}$ as function of measurement number or day (black) and the linear trend (gray).

to $850 \mathrm{kHz}$. Fig. 2 shows the resulting offsets for each day and the linear fit that shows a small upward trend. The line was obtained with a weighted least-squares fit and its uncertainty was estimated with a parametric bootstrap method. The resulting confidence interval for the slope is $(0.42 \pm 0.16)$ parts in $10^{6}$ per measurement. Even though this effect does not appear to limit our present measurement, it may become more significant for larger data sets.

Another important feature shown in Fig. 1 (b) is the $\left(a_{0}-a_{0 \text { Calc }}\right)$ offset reduction between data "set 1 " and data "set 2", which is most likely due to the magnitude of EMI coupling to the system. The $18 \mathrm{ppm}$ offset of data "set 2" is likely caused by residual EMI. Even though most of the JNT apparatus resides within the shielded room during the acquisition of data "set 2," the bit code generator (BCG) remains outside the room in order to reduce high frequency EMI. The BCG is connected to the QVNS probe head through coaxial cables, which are brought into the room via a feedthrough port.

In order to measure residual EMI coupling to the system, we performed several zero-connection measurements and also examined EMI behavior with two new QVNS chips. The new chips were made so as to provide flexible grounding options and better operating margins. Each chip has two arrays of ten $\mathrm{Nb}$ Josephson junctions with $\mathrm{Nb}_{\mathrm{x}} \mathrm{Si}_{1-\mathrm{x}}$ barriers. The new chips incorporate inner-outer DC blocks on the microwave bias lines to each array. The two different chip versions have DC blocks with cut-off frequencies of either $250 \mathrm{MHz}$ or $380 \mathrm{MHz}$.

During the EMI measurements the grounding connections were maintained in the same configuration as for data "set 2," the QVNS pulse bias was turned off, and the sense resistor was rewired to produce only uncorrelated noise. The uncorrelated noise produced by both noise sources is due to the $200 \Omega$ matching resistors [4]. Since the matching resistors for the QVNS are at $4 \mathrm{~K}$, while those for the sense resistor are at $273.16 \mathrm{~K}$, the integration time for the sense resistor EMI acquisition must be about nine times longer than that of the QVNS in order to achieve the same EMI signal to noise.

Fig. 3 shows the zero-connection cross-correlated power spectral density for the two noise sources. EMI signals are visible at $420 \mathrm{kHz}$ and $840 \mathrm{kHz}$. In order to eliminate gain effects, the mean and the standard deviation of the mean for

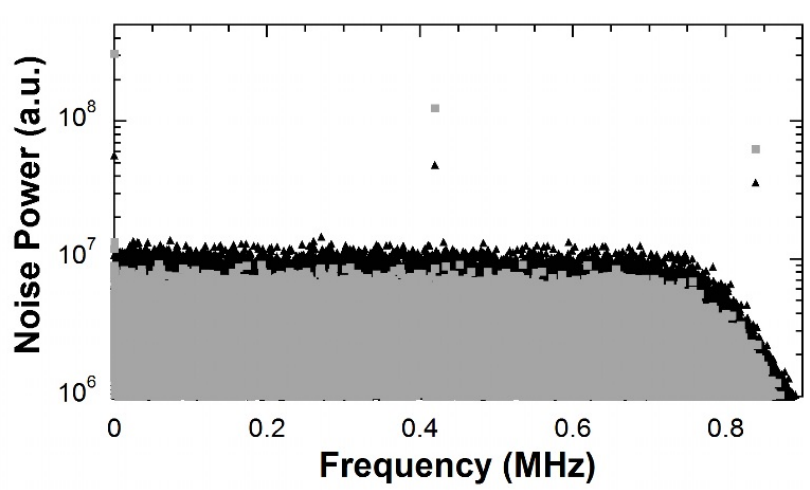

Fig. 3. Cross-correlated measured noise power spectra for the sense resistor (black) and QVNS (gray) vs. frequency. The higher points represent two EMI tones coupling to the apparatus.

both signals was calculated and normalized to the autocorrelations of the two channels. For the sense resistor we obtain a mean and relative uncertainty of $(-7.4 \pm 0.9)$ parts in $10^{6}$ when normalizing the data to the ChA auto-correlation and $(-7.7 \pm 0.9)$ normalized to $\mathrm{ChB}$. For the QVNS we have $(-5.9 \pm 2.5)$ parts in $10^{6}$ and $(-7.4 \pm 0.9)$ parts in $10^{6}$, normalized to $\mathrm{ChA}$ and $\mathrm{ChB}$, respectively. These results, which are not yet well understood, are still under investigation.

\section{CONCLUSION}

Frequency independent $\left(\mathrm{a}_{0}-\mathrm{a}_{0 \text { Calc }}\right)$ was demonstrated through a combination of new hardware and a new fitting model selection method. Preliminary EMI measurements were shown that may potentially explain the remaining offset of data "set 2," which is still under analysis.

\section{ACKNOWLEDGEMENT}

We thank John Kitching and the Time and Frequency Division for allowing us to use the shielded room for these measurements, and Paul Dresselhaus, Nathan Flower-Jacobs and Alain Rufenacht for fruitful collaborations.

\section{REFERENCES}

[1] S. P. Benz, J. M. Martinis, S. W. Nam, W. L. Tew, and D. R. White, "A new approach to Johnson noise thermometry using a Josephson quantized voltage source for calibration," Proceedings of TEMPMEKO 2001, the 8th International Symposium on Temperature and Thermal Measurements in Industry and Science, B. Fellmuth, J. Seidel, and G. Scholz, Eds., Berlin: VDE Verlag, vol. 19, pp. 37-44, April 2002.

[2] K. J. Coakley and J. Qu, "Selection of spectral distortion model for electronic determination of the Boltzmann constant," in preparation.

[3] A. Pollarolo, H. Rogalla, W. Tew, K. Coakley, and S. P. Benz "2 channels system for Boltzmann constant determination based on Johnson noise thermometry", in preparation.

[4] A. Pollarolo, T. Jeong, S. P. Benz, H. Rogalla, "Johnson noise thermometry measurement of the Boltzmann constant with a $200 \Omega$ sense resistor", IEEE Trans. Instrum. Meas., vol. 62, pp. 1512-1517, May 2013. 